\title{
Results Concerning the Analysis of Multi-Index Whittaker Function
}

\author{
Nabiullah Khan $\mathbb{D}^{1},{ }^{1}$ Saddam Husain ${ }^{(D},{ }^{2}$ Talha Usman ${ }^{(D)}{ }^{2}$ and Serkan Araci ${ }^{3}$ \\ ${ }^{1}$ Department of Applied Mathematics, Faculty of Engineering and Technology, Aligarh Muslim University, Aligarh 202002, India \\ ${ }^{2}$ Department of General Requirements, University of Technology and Applied Sciences, Sur, Oman \\ ${ }^{3}$ Department of Economics, Faculty of Economics, Administrative and Social Sciences, Hasan Kalyoncu University, \\ Gaziantep TR-27410, Turkey \\ Correspondence should be addressed to Serkan Araci; mtsrkn@gmail.com
}

Received 18 January 2022; Accepted 29 January 2022; Published 23 February 2022

Academic Editor: Clemente Cesarano

Copyright (c) 2022 Nabiullah Khan et al. This is an open access article distributed under the Creative Commons Attribution License, which permits unrestricted use, distribution, and reproduction in any medium, provided the original work is properly cited.

\begin{abstract}
A variety of functions, their extensions, and variants have been extensively investigated, mainly due to their potential applications in diverse research areas. In this paper, we aim to introduce a new extension of Whittaker function in terms of multi-index confluent hypergeometric function of first kind. We discuss multifarious properties of newly defined multi-index Whittaker function such as integral representation, integral transform (i.e., Mellin transform and Hankel transform), and derivative formula. The results presented here, being very general, are pointed out to reduce to yield some known or new formulas and identities for relatively functions.
\end{abstract}

\section{Introduction}

Generalized and multivariable forms of the special functions of mathematical physics have witnessed a significant evolution during recent years. In particular, the special functions of more than one variable provided new means of analysis for the solution of large classes of partial differential equations often encountered in physical problems. Most of the special functions of mathematical physics and their generalization have been suggested by physical problems. In mathematics, the Whittaker function is a solution of Whittaker equation, which is a modified form of confluent hypergeometric function of first kind, and it has various applications in multifarious area such as mathematical physics and many research areas, which are studied by various mathematicians (see [1-4]). Recently, many authors give an extension and generalization of several special functions such as beta function, gamma function, hypergeometric function, confluent hypergeometric function, and Whittaker function (see [2, 3, 5-12]). Ghayasuddin et al. [7] defined a new type of confluent hypergeometric function by using extended beta function in terms of multi-index Mittag-Leffler function. Inspired by the abovementioned work, in this paper, we introduced a new extension of Whittaker function in terms of multi-index Mittag-Leffler function by using an extended confluent hypergeometric function and studied their various properties such as integral transform, integral representation, and derivative formula of it. We remember below the following basic definition and extension of special function.

The classical beta function $B(u, v)$ is defined as (see [13])

$$
B(u, v)=\int_{0}^{1} t^{u-1}(1-t)^{v-1} \mathrm{~d} t=\frac{\Gamma(u) \Gamma(v)}{\Gamma(u+v)},
$$

where

$$
(\mathfrak{R}(u)>0, \mathfrak{R}(v)>0) .
$$

The classical Gauss hypergeometric function $F(u, v ; w ; \omega)$ and confluent hypergeometric function $\Phi(v ; w ; \omega)$ are defined as (see [14]) 


$$
\begin{gathered}
F(u, v ; w ; \omega)=\frac{1}{B(v, w-v)} \int_{0}^{1} t^{v-1}(1-t)^{w-v-1}(1-\omega t)^{-u} \mathrm{~d} t, \quad(|\arg (1-\omega)|<\pi ; \mathfrak{R}(w)>\mathfrak{R}(v)>0), \\
\Phi(v ; w ; \omega)=\frac{1}{B(v, w-v)} \int_{0}^{1} t^{v-1}(1-t)^{w-v-1} \exp (\omega t) \mathrm{d} t, \quad(\mathfrak{R}(w)>\mathfrak{R}(v)>0) .
\end{gathered}
$$

By using the series expansion of $(1-\omega t)^{-u}$ and $\exp (\omega t)$ in (3) and (4), respectively, the hypergeometric and confluent hypergeometric functions are written in terms of beta function as

$$
\begin{aligned}
F(u, v ; w ; \omega) & =\sum_{n=0}^{\infty}(u)_{n} \frac{B(v+n, w-v)}{B(v, w-v)} \frac{\omega^{n}}{n !}, \quad(|\omega|<1, \mathfrak{R}(w)>\mathfrak{R}(v)>0), \\
\Phi(v ; w ; \omega) & =\sum_{n=0}^{\infty} \frac{B(v+n, w-v)}{B(v, w-v)} \frac{\omega^{n}}{n !}, \quad(\mathfrak{R}(w)>\mathfrak{R}(v)>0) .
\end{aligned}
$$

In 1997, Aslam Chaudhary et al. [5] give an extension of beta function defined as

$$
B_{\rho}(u, v)=\int_{0}^{1} t^{u-1}(1-t)^{v-1} \exp \left(-\frac{\rho}{t(1-t)}\right) \mathrm{d} t
$$

where

$$
(\mathfrak{R}(\rho)>0, \mathfrak{R}(u)>0, \mathfrak{R}(v)>0) .
$$

Remark 1. If $\rho=0$, then extended beta function (6) is reduced to classical beta function (1).

In 2004, Chaudhary et al. [6] introduced the extended hypergeometric and confluent hypergeometric functions in terms of extended beta function (6) as follows:

$$
\begin{gathered}
F_{\rho}(u, v ; w ; \omega)=\sum_{n=0}^{\infty}(u)_{n} \frac{B_{\rho}(v+n, w-v)}{B(v, w-v)} \frac{\omega^{n}}{n !}, \quad(\rho \geq 0,|\omega|<1, \mathfrak{R}(w)>\Re(v)>0), \\
\Phi_{\rho}(v ; w ; \omega)=\sum_{n=0}^{\infty} \frac{B_{\rho}(v+n, w-v)}{B(v, w-v)} \frac{\omega^{n}}{n !}, \quad(\rho \geq 0, \mathfrak{R}(w)>\mathfrak{R}(v)>0) .
\end{gathered}
$$

Their integral representation is

$$
\begin{aligned}
F_{\rho}(u, v ; w ; \omega)= & \frac{1}{B(v, w-v)} \int_{0}^{1} t^{v-1}(1-t)^{w-v-1}(1-\omega t)^{-u} \exp \left(-\frac{\rho}{t(1-t)}\right) \mathrm{d} t, \\
& (\rho>0 ; \rho=0 \text { and }|\arg (1-\omega)|<\pi ; \mathfrak{R}(w)>\mathfrak{R}(v)>0), \\
\Phi_{\rho}(v ; w ; \omega)= & \frac{1}{B(v, w-v)} \int_{0}^{1} t^{v-1}(1-t)^{w-v-1} \exp \left(\omega t-\frac{\rho}{t(1-t)}\right) d t, \\
& (\rho>0 ; \rho=0 \text { and } \mathfrak{R}(w)>\mathfrak{R}(v)>0) .
\end{aligned}
$$


Shadab et al. [12] introduced an extension of beta function using generalized Mittag-Leffler function as follows:

$$
\begin{aligned}
& B_{\alpha}^{\rho}(u, v)=\int_{0}^{1} t^{u-1}(1-t)^{v-1} E_{\alpha}\left(-\frac{\rho}{t(1-t)}\right) \mathrm{d} t, \\
&\left(\mathfrak{R}(u)>0, \mathfrak{R}(v)>0, \mathfrak{R}(\rho)>0 ; \alpha \in \mathbb{R}_{0}^{+}\right),
\end{aligned}
$$

where $E_{\alpha}($.$) is the classical Mittag-Leffler (see [15,16]$ ) function defined by

$$
E_{\alpha}(\omega)=\sum_{n=0}^{\infty} \frac{\omega^{n}}{\Gamma(\alpha n+1)}
$$

where

$$
\left(\omega \in \mathbb{C}, \alpha \in \mathbb{R}_{0}^{+}\right) .
$$

Shadab et al. [12] expressed the extended hypergeometric and confluent hypergeometric functions in terms of extended beta function (11) as follows:

$$
\begin{aligned}
F_{\rho, \alpha}(u, v ; w ; \omega) & =\sum_{n=0}^{\infty}(u)_{n} \frac{B_{\alpha}^{\rho}(v+n, w-v)}{B(v, w-v)} \frac{\omega^{n}}{n !}, \quad\left(\alpha \in \mathbb{R}^{+}, \rho \in \mathbb{R}_{0}^{+},|\omega|<1, \mathfrak{R}(w)>\mathfrak{R}(v)>0\right), \\
\Phi_{\rho, \alpha}(v ; w ; \omega) & =\sum_{n=0}^{\infty} \frac{B_{\alpha}^{\rho}(v+n, w-v)}{B(v, w-v)} \frac{\omega^{n}}{n !}, \quad\left(\alpha \in \mathbb{R}^{+}, \rho \in \mathbb{R}_{0}^{+},|\omega|<1, \mathfrak{R}(w)>\mathfrak{R}(v)>0\right),
\end{aligned}
$$

and their integral representation is

$$
\begin{aligned}
F_{\rho, \alpha}(u, v ; w ; \omega)= & \frac{1}{B(v, w-v)} \times \int_{0}^{1} t^{v-1}(1-t)^{w-v-1}(1-\omega t)^{-u} E_{\alpha}\left(-\frac{\rho}{t(1-t)}\right) \mathrm{d} t \\
& \left(\alpha \in \mathbb{R}^{+}, \rho>0 ; \rho=0 \text { and }|\arg (1-\omega)|<\pi ; \Re(w)>\Re(v)>0\right), \\
\Phi_{\rho, \alpha}(v ; w ; \omega)= & \frac{1}{B(v, w-v)} \int_{0}^{1} t^{v-1}(1-t)^{w-v-1} e^{\omega t} E_{\alpha}\left(-\frac{\rho}{t(1-t)}\right) \mathrm{d} t, \\
& \left(\alpha \in \mathbb{R}^{+}, \rho>0 ; \rho=0 \text { and } \mathfrak{R}(w)>\mathfrak{R}(v)>0\right) .
\end{aligned}
$$

Ghayasuddin et al. [7] introduced an extension of beta function using multi-index Mittag-Leffler function as follows:

$$
B_{\rho}^{a_{1}, \ldots, a_{s}, b_{1}, \ldots b_{s}}(u, v)=\int_{0}^{1} t^{u-1}(1-t)^{v-1} E_{\left(1 / a_{i}\right)\left(b_{i}\right)}\left(-\frac{\rho}{t(1-t)}\right) \mathrm{d} t, \quad\left(\mathfrak{R}(u)>0, \mathfrak{R}(v)>0, \mathfrak{R}(\rho)>0 ; a_{i}>0, b_{i} \in \mathbb{R}\right),
$$

For $s=2$, if we set $1 / a_{1}=\alpha, 1 / a_{2}=0$, and $b_{1}=b_{2}=1$ in (15), then we obtain the extended beta function defined by Shadab et al. [12].

Multi-index Mittag-Leffler function $E_{\left(1 / a_{i}\right)\left(b_{i}\right)}($.$) is de-$ fined as follows (see [17]):

$$
E_{\left(1 / a_{i}\right)\left(b_{i}\right)}(\omega)=\sum_{k=0}^{\infty} \frac{\omega^{k}}{\Gamma\left(b_{1}+\left(k / a_{1}\right)\right) \ldots \Gamma\left(b_{s}+\left(k / a_{s}\right)\right)} .
$$

where $s>1$ is an integer and $a_{1}, a_{2}, \ldots, a_{s}>0$ and $b_{1}, b_{2}, \ldots, b_{s}$ are arbitrary real numbers.

It is easy to see that, for $s=2,\left(1 / a_{1}\right)=\alpha,\left(1 / a_{2}\right)=0$, and $b_{1}=b_{2}=1$, then multi-index Mittag-Leffler function is reduced to classical Mittag-Leffler function $E_{\alpha}(\omega)$.

In 2020, Ghayasuddin et al. [7] expressed the extended hypergeometric and confluent hypergeometric functions in terms of extended beta function (15) as follows: 


$$
\begin{gathered}
F_{\rho}^{a_{1}, \ldots, a_{s}, b_{1}, \ldots, b_{s}}(u, v ; w ; \omega)=\sum_{n=0}^{\infty}(u)_{n} \frac{B_{\rho}^{a_{1}, \ldots, a_{s}, b_{1}, \ldots, b_{s}}(v+n, w-v)}{B(v, w-v)} \frac{\omega^{n}}{n !}, \quad\left(a_{i}>0, b_{i} \in \mathbb{R}, \rho \in \mathbb{R}_{0}^{+},|\omega|<1, \mathfrak{R}(w)>\mathfrak{R}(v)>0\right), \\
\Phi_{\rho}^{a_{1}, \ldots, a_{s}, b_{1}, \ldots, b_{s}}(v ; w ; \omega)=\sum_{n=0}^{\infty} \frac{B_{\rho}^{a_{1}, \ldots, a_{s}, b_{1}, \ldots, b_{s}}(v+n, w-v)}{B(v, w-v)} \frac{\omega^{n}}{n !}, \quad\left(a_{i}>0, b_{i} \in \mathbb{R}, \rho \in \mathbb{R}_{0}^{+},|\omega|<1, \mathfrak{R}(w)>\mathfrak{R}(v)>0\right),
\end{gathered}
$$

and their integral representation is

$$
\begin{aligned}
F_{\rho}^{a_{1}, \ldots, a_{s}, b_{1}, \ldots, b_{s}}(u, v ; w ; \omega)= & \frac{1}{B(v, w-v)} \int_{0}^{1} t^{v-1}(1-t)^{w-v-1}(1-\omega t)^{-u} E_{\left(1 / a_{i}\right)\left(b_{i}\right)}\left(-\frac{\rho}{t(1-t)}\right) \mathrm{d} t, \\
& \left(\rho>0 ; \rho=0 \text { and }|\arg (1-\omega)|<\pi ; \Re(w)>\Re(v)>0, a_{i}>0, b_{i} \in \mathbb{R}\right), \\
\Phi_{\rho}^{a_{1}, \ldots, a_{s}, b_{1}, \ldots, b_{s}}(v ; w ; \omega)= & \frac{1}{B(v, w-v)} \times \int_{0}^{1} t^{v-1}(1-t)^{w-v-1} e^{\omega t} E_{\left(1 / a_{i}\right)\left(b_{i}\right)}\left(-\frac{\rho}{t(1-t)}\right) \mathrm{d} t, \\
& \left(\rho>0 ; \rho=0 \text { and } \mathfrak{R}(w)>\Re(v)>0, a_{i}>0, b_{i} \in \mathbb{R}\right) .
\end{aligned}
$$

The extension of Kummer's relation to the generalized extended confluent hypergeometric function of the first kind is as follows:

$$
\Phi_{\alpha, \beta}^{\rho, \eta, v}(v, w ; \omega)=e^{\omega} \Phi_{\alpha, \beta}^{\rho, \eta, v}(w-v ; w ;-\omega) .
$$

For $\alpha=\beta=\eta=\nu=1$ and $\rho=0$, (20) is reduced to Kummer's formula of first kind for the classical confluent hypergeometric function (see [14]).

The Whittaker function $M_{\kappa, \zeta}(\omega)$ in terms of confluent hypergeometric function of first kind (see $[4,18]$ ) is defined as

$$
M_{\kappa, \zeta}(\omega)=\omega^{\zeta+(1 / 2)} \exp \left(\frac{-\omega}{2}\right) \Phi\left(\zeta-\kappa+\frac{1}{2} ; 2 \zeta+1 ; \omega\right), \quad\left(\mathfrak{R}(\zeta)>\frac{-1}{2} \text { and } \mathfrak{R}(\zeta \pm \kappa)>\frac{-1}{2}\right)
$$

In 2013, Nagar et al. [3] generalized the Whittaker function by using extended confluent hypergeometric function $\Phi_{\rho}$ which is defined as

$$
M_{\rho, \kappa, \zeta}(\omega)=\omega^{\zeta+(1 / 2)} \exp \left(\frac{-\omega}{2}\right) \Phi_{\rho}\left(\zeta-\kappa+\frac{1}{2} ; 2 \zeta+1 ; \omega\right), \quad\left(\Re(\zeta)>\frac{-1}{2} \text { and } \Re(\zeta \pm \kappa)>\frac{-1}{2}\right)
$$

\section{Multi-Index Whittaker Function}

In this section, we give a new generalization of Whittaker function of the first kind by applying the multi-index confluent hypergeometric function (19) defined as

$$
M_{\rho, \kappa, \zeta}^{a_{1}, \ldots, a_{s}, b_{1}, \ldots, b_{s}}(\omega)=\omega^{\zeta+1 / 2} \exp \left(\frac{-\omega}{2}\right) \Phi_{\rho}^{a_{1}, \ldots, a_{s}, b_{1}, \ldots, b_{s}}\left(\zeta-\kappa+\frac{1}{2} ; 2 \zeta+1 ; \omega\right), \quad\left(\rho \geq 0, \Re(\zeta)>\frac{-1}{2} \text { and } \Re(\zeta \pm \kappa)>\frac{-1}{2}, a_{i}>0, b_{i} \in \mathbb{R}\right)
$$

If we take $\rho=0$ and $a_{i}=\cdots=a_{s}=b_{i}=\cdots=b_{s}=1,(23)$ reduced to the classical Whittaker function (21). 
2.1. Integral Representation. Here, we define integral representation of the multi-index Whittaker function by using (19) and (23) as

$$
\begin{aligned}
M_{\rho, \kappa, \zeta}^{a_{1}, \ldots, a_{s}, b_{1}, \ldots, b_{s}}(\omega)= & \frac{\omega^{\zeta+1 / 2} \exp (-\omega / 2)}{B(\zeta-\kappa+1 / 2, \zeta+\kappa+1 / 2)} \times \int_{0}^{1} t^{\zeta-\kappa-1 / 2}(1-t)^{\zeta+\kappa-1 / 2} e^{\omega t} E_{\left(1 / a_{i}\right)\left(b_{i}\right)}\left(-\frac{\rho}{t(1-t)}\right) \mathrm{d} t \\
& \cdot\left(\rho \geq 0, \mathfrak{R}(\zeta)>\frac{-1}{2} \text { and } \mathfrak{R}(\zeta \pm \kappa)>\frac{-1}{2}, a_{i}>0, b_{i} \in \mathbb{R}\right) .
\end{aligned}
$$

Substituting $t=(x-a) /(b-a)$ in $(24)$, we get the multiindex Whittaker function as

$$
\begin{aligned}
M_{\rho, \kappa, \zeta}^{a_{1}, \ldots, a_{s}, b_{1}, \ldots, b_{s}}(\omega)= & \frac{(b-a)^{-2 \zeta+1} \omega^{\zeta+(1 / 2)} \exp (-\omega / 2)}{B(\zeta-\kappa+(1 / 2), \zeta+\kappa+(1 / 2))} \\
& \times \int_{a}^{b}(x-a)^{\zeta-\kappa-(1 / 2)}(b-x)^{\zeta+\kappa-(1 / 2)} e^{\omega(x-a / b-a)} E_{\left(1 / a_{i}\right)\left(b_{i}\right)}\left(-\frac{\rho(b-a)^{2}}{(x-a)(b-x)}\right) \mathrm{d} x
\end{aligned}
$$

where a and $b$ are scalars such that $(b-a)>0$.

If we take $a=-1$ and $b=1$ in (23), we obtain another representation of multi-index Whittaker function:

$M_{\rho, \kappa, \zeta}^{a_{1}, \ldots, a_{s}, b_{1}, \ldots, b_{s}}(\omega)=\frac{2^{-2 \zeta+1} \omega^{\zeta+(1 / 2)}}{B(\zeta-\kappa+(1 / 2), \zeta+\kappa+(1 / 2))} \times \int_{a}^{b}(x+1)^{\zeta-\kappa-(1 / 2)}(1-x)^{\zeta+\kappa-(1 / 2)} e^{(\omega x / 2)} E_{\left(1 / a_{i}\right)\left(b_{i}\right)}\left(-\frac{4 \rho}{(x+1)(1-x)}\right) \mathrm{d} x$,

In (24), substitute $t=x /(1+x)$, and we get another integral representation of multi-index Whittaker function as

$$
\begin{aligned}
& M_{\rho, \kappa, \zeta}^{a_{1}, \ldots, a_{s}, b_{1}, \ldots, b_{s}}(\omega)= \frac{\omega^{\zeta+(1 / 2)} \exp (-\omega / 2)}{B(\zeta-\kappa+(1 / 2), \zeta+\kappa+(1 / 2))} \\
& \times \int_{0}^{\infty} x^{\zeta-\kappa-(1 / 2)}(1+x)^{-(2 \zeta+1)} e^{\omega x /(1+x)} E_{\left(1 / a_{i}\right)\left(b_{i}\right)}\left(-\frac{\rho(1+x)^{2}}{x}\right) \mathrm{d} x \\
&=b_{i}=\ldots=b_{s}=1 \text { and } \rho=0 \text { in }(24),(23), \quad M_{\rho, \kappa, \zeta}^{a_{1}, \ldots a_{s}, b_{1}, \ldots b_{s}}(-\omega)=(-1)^{\zeta+(1 / 2)} M_{\rho, \eta, \nu}^{a_{1}, \ldots, a_{s}, b_{1}, \ldots, b_{s}}(\omega), \quad(\rho \geq 0)
\end{aligned}
$$

If $a_{i}=\cdots=a_{s}=b_{i}=\cdots=b_{s}=1$ and $\rho=0$ in (24), (23), and (27), we obtain integral representation of classical Whittaker function.

Theorem 1. The following relation holds true:

Proof. Using relation (20) in (23), we have

$$
M_{\rho, \kappa, \zeta}^{a_{1}, \ldots a_{s}, b_{1}, \ldots b_{s}}(-\omega)=(-1)^{\zeta+(1 / 2)} \omega^{\zeta+(1 / 2)} \exp \left(\frac{-\omega}{2}\right) \Phi_{\rho}^{a_{1}, \ldots, a_{s}, b_{1}, \ldots, b_{s}}\left(\zeta-\kappa+\frac{1}{2} ; 2 \zeta+1 ; \omega\right) .
$$


Now, writing the right-hand side of the above representation by using (23), we get the desired result.

$$
\begin{aligned}
\int_{0}^{\infty} \rho^{s-1} M_{\rho, \kappa, \zeta}^{a_{1}, \ldots, a_{s}, b_{1}, \ldots, b_{s}}(\omega) \mathrm{d} \rho \\
=\frac{\Gamma(s) \Gamma(1-s)}{\prod_{i=0}^{m} \Gamma\left(b_{i}-\left(s / a_{i}\right)\right)} \frac{B((\zeta+s)-\kappa+(1 / 2), 2(\zeta+s)+1)}{\omega^{s} B(\zeta-\kappa+(1 / 2), \zeta+\kappa+(1 / 2))} M_{\kappa, \zeta+s}(\omega) \\
\\
\quad \cdot\left(\mathfrak{R}(s)>0 \text { and } \Re(\zeta \pm \kappa)>\frac{-1}{2}, a_{i}>0, b_{i} \in \mathbb{R}\right) .
\end{aligned}
$$

Proof. Using multi-index Whittaker function (1), we obtain

$$
\int_{0}^{\infty} \rho^{s-1} M_{\rho, \kappa, \zeta}^{a_{1}, \ldots, a_{s}, b_{1}, \ldots, b_{s}}(\omega) \mathrm{d} \rho=\omega^{\zeta+(1 / 2)} \exp \left(\frac{-\omega}{2}\right) \int_{0}^{\infty} \rho^{s-1} \Phi_{\rho}^{a_{1}, \ldots, a_{s}, b_{1}, \ldots, b_{s}}\left(\zeta-\kappa+\frac{1}{2} ; 2 \zeta+1 ; \omega\right) \mathrm{d} \rho
$$

Again, using multi-index confluent hypergeometric function (19), we obtain

$$
=\frac{\omega^{\zeta+(1 / 2)} \exp (-\omega / 2)}{B(\zeta-\kappa+(1 / 2), \zeta+\kappa+(1 / 2))} \times \int_{0}^{\infty} \rho^{s-1} \int_{0}^{1} t^{\zeta-\kappa-(1 / 2)}(1-t)^{\zeta+\kappa-(1 / 2)} e^{\omega t} E_{\left(1 / a_{i}\right)\left(b_{i}\right)}\left(-\frac{\rho}{t(1-t)}\right) \mathrm{d} t \mathrm{~d} \rho
$$

Changing the order of integration, we obtain

$$
=\frac{\omega^{\zeta+(1 / 2)} \exp (-\omega / 2)}{B(\zeta-\kappa+(1 / 2), \zeta+\kappa+(1 / 2))} \times \int_{0}^{1} t^{\zeta-\kappa-(1 / 2)}(1-t)^{\zeta+\kappa-(1 / 2)} e^{\omega t} \int_{0}^{\infty} \rho^{s-1} E_{\left(1 / a_{i}\right)\left(b_{i}\right)}\left(-\frac{\rho}{t(1-t)}\right) \mathrm{d} \rho \mathrm{d} t
$$

Substituting $u=\rho /(t(t-1))$ in integral (33), we obtain

$$
\begin{aligned}
= & \frac{\omega^{\zeta+(1 / 2)} \exp (-\omega / 2)}{B(\zeta-\kappa+(1 / 2), \zeta+\kappa+(1 / 2))} \\
& \times \int_{0}^{1} t^{\zeta+s-\kappa-(1 / 2)}(1-t)^{\zeta+s+\kappa-(1 / 2)} e^{\omega t} \int_{0}^{\infty} u^{s-1} E_{\left(1 / a_{i}\right)\left(b_{i}\right)}(-u) \mathrm{d} u \mathrm{~d} t .
\end{aligned}
$$

Now, using well-known result (p. 102 of [19]) and confluent hypergeometric function (4), we obtain 


$$
\begin{aligned}
= & \frac{\omega^{\zeta+(1 / 2)} \exp (-\omega / 2) B((\zeta+s)-\kappa+(1 / 2), 2(\zeta+s)+1)}{B(\zeta-\kappa+(1 / 2), \zeta+\kappa+(1 / 2))} \\
& \times \phi(\zeta+s-\kappa+(1 / 2) ; 2(\zeta+s)+1 ; \omega) \frac{\Gamma(s) \Gamma(1-s)}{\prod_{i=0}^{m} \Gamma\left(b_{i}-\left(s / a_{i}\right)\right)} \\
= & \frac{\omega^{\zeta+s+(1 / 2)} \exp (-\omega / 2) \phi((\zeta+s)-\kappa+(1 / 2) ; 2(\zeta+s)+1 ; \omega)}{\omega^{s} B(\zeta-\kappa+(1 / 2), \zeta+\kappa+(1 / 2))} \\
& \times B\left((\zeta+s)-\kappa+\frac{1}{2}, 2(\zeta+s)+1\right) \frac{\Gamma(s) \Gamma(1-s)}{\prod_{i=0}^{m} \Gamma\left(b_{i}-\left(s / a_{i}\right)\right)} .
\end{aligned}
$$

By using definition of classical Whittaker function (21), we get the desired result.

$$
\begin{aligned}
\int_{0}^{\infty} \omega^{a-1} e^{-b \omega} M_{\rho, \kappa, \zeta}^{a_{1}, \ldots, a_{s}, b_{1}, \ldots b_{s}}(c \omega) \mathrm{d} \omega \\
=\frac{c^{\zeta+(1 / 2)} \Gamma(a+\zeta+(1 / 2))}{(b+(c / 2))^{a+\zeta+(1 / 2)}} F_{\rho}^{a_{1}, \ldots, a_{s}, b_{1}, \ldots, b_{s}}\left(a+\zeta+\frac{1}{2}, \zeta-\kappa+\frac{1}{2} ; 2 \zeta+1 ; \frac{2 c}{2 b+c}\right) \\
\quad \cdot\left(\rho \geq 0,2 b>c>0, \Re(a+\zeta)>\frac{-1}{2}, a_{i}>0, b_{i} \in \mathbb{R}\right) .
\end{aligned}
$$

Proof. Using integral representation of the multi-index

Whittaker function, we obtain

$$
\begin{aligned}
\int_{0}^{\infty} & \omega^{a-1} e^{-b \omega} M_{\rho, \kappa, \zeta}^{a_{1}, \ldots, a_{s}, b_{1}, \ldots, b_{s}}(c \omega) \mathrm{d} \omega \\
= & \frac{1}{B(\zeta-\kappa+(1 / 2), \zeta+\kappa+(1 / 2))} \int_{0}^{\infty} \omega^{a-1} e^{-b \omega}(c \omega)^{\zeta+(1 / 2)} \exp \left(\frac{-c \omega}{2}\right) \\
& \times \int_{0}^{1} t^{\zeta-\kappa-(1 / 2)}(1-t)^{\zeta+\kappa-(1 / 2)} e^{c \omega t} E_{\left(1 / a_{i}\right)\left(b_{i}\right)}\left(-\frac{\rho}{t(1-t)}\right) \mathrm{d} t \mathrm{~d} \omega
\end{aligned}
$$

Now, interchanging the order of integration and using the definition of gamma function, we obtain

$$
\begin{aligned}
\int_{0}^{\infty} & \omega^{a-1} e^{-b \omega} M_{\rho, \kappa, \zeta}^{a_{1}, \ldots, a_{s}, b_{1}, \ldots, b_{s}}(c \omega) \mathrm{d} \omega \\
= & \frac{c^{\zeta+(1 / 2)} \Gamma(a+\zeta+(1 / 2))}{(b+(c / 2))^{a+\zeta+(1 / 2)} B(\zeta-\kappa+(1 / 2), \zeta+\kappa+(1 / 2))} \\
& \times \int_{0}^{1} t^{\zeta-\kappa-(1 / 2)}(1-t)^{\zeta+\kappa-(1 / 2)}\left(1-\frac{2 c t}{2 b+c}\right)^{-(a+\zeta+(1 / 2))} E_{\left(1 / a_{i}\right)\left(b_{i}\right)}\left(-\frac{\rho}{t(1-t)}\right) \mathrm{d} t \mathrm{~d} \omega
\end{aligned}
$$


Using (18) in (38), we get the desired result (36).
Corollary 1. If we take $a=c=1$ in (36), we get the following special cases:

$$
\begin{aligned}
& \int_{0}^{\infty} e^{-b \omega} M_{\rho, \kappa, \zeta}^{a_{1}, \ldots a_{s}, b_{1}, \ldots b_{s}}(\omega) \mathrm{d} \omega \\
& \quad=\frac{2^{\zeta+(3 / 2)} \Gamma(\zeta+(3 / 2))}{(2 b+1)^{\zeta+(3 / 2)}} F_{\rho}^{a_{1}, \ldots, a_{s}, b_{1}, \ldots, b_{s}}\left(\zeta+\frac{3}{2}, \zeta-\kappa+\frac{1}{2} ; 2 \zeta+1 ; \frac{2}{2 b+c}\right) .
\end{aligned}
$$

Theorem 4. The following Hankel transform formula holds

true:

$$
\begin{aligned}
\int_{0}^{\infty} & \omega M_{\rho, \kappa, \zeta}^{a_{1}, \ldots, a_{s}, b_{1}, \ldots, b_{s}}(\omega) J_{m}(a \omega) \mathrm{d} \omega \\
= & \frac{\Gamma(\zeta+\kappa+(5 / 2))}{\left(a^{2}+(1 / 4)\right)^{(\zeta / 2)+(5 / 4)}} \sum_{n=o}^{\infty} \frac{B_{\rho}^{a_{1}, \ldots, a_{s}, b_{1}, \ldots, b_{s}}(\zeta-\kappa+n+(1 / 2), \zeta+\kappa+(1 / 2))(\zeta+m+(5 / 2))_{n}}{B(\zeta-\kappa+(1 / 2), \zeta+\kappa+(1 / 2))\left(a^{2}+(1 / 4)\right)^{n / 2} n !} \\
& \times P_{\zeta+n+(3 / 2)}^{-m}\left(\frac{1}{\sqrt{4 a^{2}+1}}\right),
\end{aligned}
$$$$
\left(\mathfrak{R}(\zeta \pm \kappa)>\frac{-1}{2} \text { and } \Re(\zeta+m)>\frac{-5}{2}, a_{i}>0, b_{i} \in \mathbb{R}\right),
$$

Proof. Using (23) and (18), expanding multi-index Whittaker function in terms of generating extended beta function and changing the order of integration and summation, we obtain

where $P_{\zeta}^{m}(z)$ is the Legendre function (see p.34. of [20]).

$$
\begin{aligned}
\int_{0}^{\infty} z M_{\rho, \kappa, \zeta}^{a_{1}, \ldots, a_{s}, b_{1}, \ldots, b_{s}}(\omega) J_{m}(a \omega) \mathrm{d} \omega= & \sum_{n=o}^{\infty} \frac{B_{\rho}^{a_{1}, \ldots, a_{s}, b_{1}, \ldots, b_{s}}(\zeta-\kappa+n+(1 / 2), \zeta+\kappa+(1 / 2))}{B(\zeta-\kappa+(1 / 2), \zeta+\kappa+(1 / 2)) n !} \\
& \times \int_{0}^{\infty} \omega^{\zeta+n+(3 / 2)} e^{-\omega / 2} J_{m}(a \omega) \mathrm{d} \omega .
\end{aligned}
$$

On using the known result (see p. 182 (9)of[21]),

$$
\int_{0}^{\infty} e^{-p t} t^{\zeta} J_{m}(a t) \mathrm{d} t=\Gamma(\zeta+m+1) r^{-\zeta-1} P_{\zeta}^{-m}\left(\frac{p}{r}\right),
$$

where $\mathfrak{R}(\zeta+m)>-1, r=\left(p^{2}+a^{2}\right)^{1 / 2}$ and $P_{\zeta}^{m}(z)$ is the Legendre function (see [20]).By using (43) in (42) and after some simplification, we get the desired result.

\section{Derivative of Multi-Index Whittaker Function}

Theorem 5. The following differential formula holds true:

$$
\frac{\mathrm{d}^{n}}{\mathrm{~d} \omega^{n}}\left[e^{\omega / 2} \omega^{-\zeta-(1 / 2)} M_{\rho, \kappa, \zeta}^{a_{1}, \ldots, a_{s}, b_{1}, \ldots, b_{s}}(\omega)\right]=\frac{(\zeta-\kappa+(1 / 2))_{n}}{(2 \zeta+1)_{n}} e^{\omega / 2} \omega^{-\zeta-(n / 2)-(1 / 2)} M_{\rho, \kappa}-\frac{n}{2}, \zeta+\frac{n^{a_{1}, \ldots, a_{s}, b_{1}, \ldots, b_{s}}}{2}(\omega)
$$


Proof. The $n^{\text {th }}$ order derivative of generalized extended confluent hypergeometric function is given by [9]

$$
\begin{aligned}
& \frac{\mathrm{d}^{n}}{\mathrm{~d} \omega^{n}}\left[\Phi_{\rho}^{a_{1}, \ldots, a_{s}, b_{1}, \ldots, b_{s}}(v ; w ; \omega)\right] \\
& \quad=\frac{(v)_{n}}{(w)_{n}} \Phi_{\rho}^{a_{1}, \ldots, a_{s}, b_{1}, \ldots, b_{s}}(v+n ; w+n ; \omega) .
\end{aligned}
$$

Now, using (23) on the left-hand side of (44), we obtain

$$
\begin{aligned}
& \frac{\mathrm{d}^{n}}{\mathrm{~d} \omega^{n}}\left[e^{\omega / 2} z^{\left.-\zeta-(1 / 2)^{a_{1}, \ldots, a_{s}, b_{1}, \ldots, b_{s}}(\omega)\right]}\right. \\
& \quad=\frac{\mathrm{d}^{n}}{\mathrm{~d} \omega^{n}}\left[\Phi_{\rho}^{a_{1}, \ldots, a_{s}, b_{1}, \ldots, b_{s}}\left(\zeta-\kappa+\frac{1}{2} ; 2 \zeta+1 ; \omega\right)\right] \\
& \quad=\frac{(\zeta-\kappa+(1 / 2))_{n}}{(2 \zeta+1)_{n}} \times \Phi_{\rho}^{a_{1}, \ldots, a_{s}, b_{1}, \ldots, b_{s}}\left[\left(\zeta+\frac{n}{2}\right)-\left(\kappa-\frac{n}{2}\right)+\frac{1}{2} ; 2\left(\zeta+\frac{1}{2}\right)+1 ; \omega\right] \\
& =\frac{(\zeta-\kappa+(1 / 2))_{n}}{(2 \zeta+1)_{n}} e^{\omega / 2} \omega^{-\zeta-(n / 2)-(1 / 2)} M_{\rho, \kappa-(n / 2), \zeta+(n / 2)}^{a_{1}, \ldots a_{s}, b_{1}, \ldots, b_{s}}(\omega) .
\end{aligned}
$$

We get the desired result (44).

\section{Conclusion}

In the present paper, we introduce a multi-index Whittaker function in terms of extended confluent hypergeometric function. We have provided some important properties of Whittaker function such as integral representation, integral transform, and derivative formula. We have known that most of the special function of mathematical physics such as modified Bessel function and Laguerre and Hermite polynomial can be written in terms of Whittaker function. Therefore, extensions and generalization of the Whittaker function are playing important roles in applied mathematics and mathematical physics.

\section{Data Availability}

No data were used to support this study.

\section{Conflicts of Interest}

The authors declare that they have no conflicts of interest.

\section{References}

[1] M. Ali, M. Ghayasuddin, and N. U. Khan, "Certain new extension of Whittaker function and its properties," Indian Journal of Mathematics, vol. 2, no. 1, pp. 81-96, 2010.

[2] N. Khan and M. Ghayasuddin, "A note on generalized extended Whittaker function," Honam Mathematical Journal, vol. 38, no. 2, pp. 325-335, 2016.

[3] D. K. Nagar, R. A. M. Vasquez, and A. K. Gupta, "Properties of extended Whittaker function," Journal. Progress in Applied Mathematics, vol. 6, no. 2, pp. 70-80, 2013.

[4] E. T. Whittaker and G. N. Watson, A Course of Mordern Analysis, Reprint of the 4th ed. Cambridge Mathematical
Library, Cambridge University Press, Cambridge, UK, 1990.

[5] M. Aslam Chaudhry, A. Qadir, M. Rafique, and S. M. Zubair, "Extension of Euler's beta function," Journal of Computational and Applied Mathematics, vol. 78, no. 1, pp. 19-32, 1997.

[6] M. A. Chaudhry, A. Qadir, H. M. Srivastava, and R. B. Paris, "Extended hypergeometric and confluent hypergeometric functions," Applied Mathematics and Computation, vol. 159, no. 2, pp. 589-602, 2004.

[7] M. Ghayasuddin, N. Khan, and M. Ali, "A Study of extended beta, Gauss and confluent hypergeometric functions," International Journal of Apllied Mathematics, vol. 33, no. 1, pp. 1-13, 2020.

[8] N. U. Khan and S. Husain, "A note on extended beta function inolving generalized Mittag-Leffler function and its applications," TWMS Journal of Applied and Engineering Mathematics, (Accepted), 2020.

[9] D. M. Lee, A. K. Rathie, R. K. Parmar, and Y. S. Kim, "Generalization of extended beta function, hypergeometric and confluent hypergeometric functions," Honam Mathematical Journal, vol. 33, no. no. 2, pp. 187-206, 2011.

[10] E. Özergin, M. A. Özarslan, and A. Altın, "Extension of gamma, beta and hypergeometric functions," Journal of Computational and Applied Mathematics, vol. 235, no. 16, pp. 4601-4610, 2011.

[11] R. K. Parmar, "A new generalization of Gamma, Beta, hypergeometric andconfluent hypergeometric functions," Le Matematiche, vol. LXVIII, pp. 33-52, 2013.

[12] M. Shadab, S. Jabee, and J. Choi, "An extended beta function and its applications," Far East Journal of Mathematical Sciences, vol. 103, no. 1, pp. 235-251, 2018.

[13] G. E. Andrews, R. Askey, and R. Roy, Special Functions, Cambridge University Press, Cambridge, UK, 1999.

[14] E. D. Rainville, Special Functions, The Macmillan Company, New York, NY, USA, 1960.

[15] G. M. Mittag-Leffler, "Sur la nouvelle function $E_{\alpha}(x)$," Comptes Rendus de l'Académie des Sciences, vol. 137, pp. 554-558, 1903. 
[16] G. Mittag-Leffler, "Sur la représentation analytique d'une branche uniforme d'une fonction monogène: cinquième note," Acta Mathematica, vol. 29, pp. 101-181, 1905.

[17] V. Kiryakova, "Multiindex Mittag-Leffler functions, related Gelfond-Leontiev operators and Laplace type integral transforms," Fractional Calculus and Applied Analysis, vol. 2, no. 4, pp. 445-462, 1999.

[18] E. T. Whittaker, "An expression of certain known functions as generalized hypergeometric functions," Bulletin of the American Mathematical Society, vol. 10, no. 3, pp. 125-134, 1903.

[19] R. Gorenflo, A. A. Kilbas, F. Mainardi, and S. V. Rogosin, Mittag-Leffler Functions, Related Topics and Applications, Springer, Berlin, Germany, 2014.

[20] H. M. Srivastava and H. L. Manocha, A Tretise on Generating Functions, Halsted Press (Ellis Horwood Limited, Chichester), John Wiley and Sons, New York, NY, USA, 1984.

[21] A. Erdelyi, W. Magnus, F. Oberhettinger, and F. G. Tricomi, Tables of Integral Transform, Vol I, McGraw-Hill, New York, NY, USA, 1954. 